\title{
Changes of gene expression in T lymphocytes following Golli-MBP gene RNA interference
}

\author{
JUANYONG XU ${ }^{1,2^{*}}$, DANDAN ZHU ${ }^{1,2^{*}}$, JING SHAN $^{1,2}$ and YUAN FAN ${ }^{1,2}$ \\ ${ }^{1}$ Jiangsu Key Laboratory of Oral Diseases; ${ }^{2}$ Department of Oral Medicine, Affiliated Hospital of Stomatology, \\ Nanjing Medical University, Nanjing, Jiangsu 210029, P.R. China
}

Received September 21, 2015; Accepted September 26, 2016

DOI: $10.3892 / \mathrm{mmr} .2016 .5850$

\begin{abstract}
The major cell types expressing Golli in the immune system are the T-lineage cells. The aim of the current study was to investigate the changes of gene expression in T lymphocytes subsequent to downregulation of the Golli-myelin basic protein (MBP) gene. RNA interference technology was used to suppress the expression of Golli-MBP in Jurkat cells and DNA microarray techniques were applied to investigate the alterations of gene expression profiles. The results indicated that there were 387 differentially expressed genes. In the Golli-MBP knockdown Jurkat cells, the expression of 108 genes was enhanced, 279 genes were suppressed. Gene ontology analysis identified differentially expressed genes involved in several biological progresses, including cell adhesion and immune responses. Pathway analysis demonstrated that the majority of the differentially expressed genes $(23.3 \%)$ were involved in cytokine-cytokine receptor interaction. Subsequent to Golli-MBP knockdown, the mechanisms that changed the biological characteristics of Jurkat cells were complex, involving numerous types of functional proteins, and metabolic and signaling pathways. However, further experiments are required to confirm these results.
\end{abstract}

\section{Introduction}

The myelin basic protein (MBP) gene is a genetic complex containing three transcription start sites, which generate two families of proteins. Golli proteins are generated from the first transcription start site, while the classic MBPs are produced from the second and third transcription start sites. The classic MBPs are major constituents of the myelin sheath of oligodendrocytes and Schwann cells in the central and peripheral nervous system,

Correspondence to: Professor Yuan Fan, Jiangsu Key Laboratory of Oral Diseases, Nanjing Medical University, 136 Hanzhong Road, Nanjing, Jiangsu 210029, P.R. China

E-mail: fanyuan65@hotmail.com

*Contributed equally

Key words: Golli-MBP, T lymphocyte, chemokine, DNA microarray, cytokine respectively. The Golli-MBPs are structurally associated with the classic MBPs, however are more ubiquitously expressed, particularly in the immune system, including $\mathrm{T}$ lymphocytes, B lymphocytes and macrophages (1-3). T lymphocytes serve an important role in the immune system. Numerous diseases of the immune system are associated with the proliferation and apoptosis of T lymphocytes. The study of T lymphocytes may aid in the clarification of the pathogenesis of immune diseases. Golli-MBP is predominantly expressed in T cells within the immune system, which can negatively regulate the proliferation and activation of the $\mathrm{T}$ cells through a mechanism involving the modulation of calcium homeostasis $(4,5)$. Thus, Golli-MBP may be an important pathogenetic factor associated with immune diseases.

In the current study, a Golli-MBP-RNAi lentiviral vector was constructed and it was used to transfect Jurkat cells. DNA microarray techniques were applied to investigate alterations in gene expression profiles. DNA microarrays measure the expression levels of large numbers of genes simultaneously. With the extensive use of microarrays, expression profiling has identified numerous applications including the discovery of gene functions and pathway dissection. In the current study, the Agilent human gene microarray system was used, which may aid in determining the detailed functional roles of numerous associated genes in the development of autoimmune diseases.

\section{Materials and methods}

Materials. The T lymphocytic leukemia Jurkat cell line was purchased from the cell bank of the Chinese Academy of Sciences (Shanghai, China). Three samples from Golli-MBP knockdown Jurkat cells and three samples from control Jurkat cells were included in the study. The Agilent Human Gene Expression system (8*60K; design ID: 039494; Agilent Technologies, Inc., Santa Clara, CA, USA) was used in the current study, and data analysis of the 6 samples was completed.

Lentiviral vector preparation and transfection. RNAi was used for silencing Golli-MBP in the Jurkat cells. The target sequence was 5'-GGGAGGACAACACCTTCAA-3'. The sense strand of the oligonucleotide sequence was 5'-CCGGGAGGGAGG ACAACACCTTCAACTCGAGTTGAAGGTGTTGTCCTC CCTCTTTTTG-3' and the antisense strand was 5'-AATTCA AAAAGAGGGAGGACAACACCTTCAACTCGAGTTGAA 
GGTGTTGTCCTCCCTC-3'. The Golli-MBP-RNAi lentiviral vector (GV248-KD-MBP) labeled with green fluorescent protein was prepared by GeneChem Co., Ltd. (Shanghai, China). Jurkat cells were cultured in RPMI- 1640 medium at $37^{\circ} \mathrm{C}$ with $5 \% \mathrm{CO}_{2}$ and $30 \%$ saturated humidity. The medium was supplemented with $10 \%$ fetal calf serum, $100 \mathrm{IU} / \mathrm{ml}$ penicillin and $100 \mathrm{IU} / \mathrm{ml}$ streptomycin (Gibco; Thermo Fisher Scientific, Inc., Waltham, MA, USA). Jurkat cells in the logarithmic growth phase were seeded at a density of $1.5 \times 10^{7}$ cells/well in six-well plates and were transfected with GV248-KD-MBP, which also included a blank control and a negative control. The multiplicity of infection ratio of 80 was selected, in addition, $8 \mu \mathrm{g} / \mathrm{ml}$ polybrene (Takara Bio, Inc., Otsu, Japan) was added to increase infection efficiencies. Subsequent to $72 \mathrm{~h}$ incubation, Jurkat cells were observed under an inverted fluorescence microscope and cells with $>80 \%$ transfection efficiency were considered for gene interference.

RNA extraction. Total RNA extraction was performed using a Qiagen RNeasy Mini Kit (Qiagen China Co., Ltd., Shanghai, China) according to the manufacturer's instructions. Total RNA was quantified using NanoDrop ND-2000 (Thermo Fisher Scientific, Inc.) and the RNA integrity was assessed using an Agilent Bioanalyzer 2100 (Agilent Technologies, Inc.). RNA samples with RIN $\geq 7.0$ and $28 \mathrm{~S} / 18 \mathrm{~S}>0.7$ were used. All equipment (homogenizers, mortar, pestle etc.) was pretreated with RNase and rinsed with diethyl pyrocarbonate-treated water prior to use.

Preparation of fluorescent cDNA probes. Double-stranded cDNA was synthesized from total RNA using the SuperScript Double-Stranded cDNA Synthesis kit (Invitrogen; Thermo Fisher Scientific, Inc.). cRNA was then obtained from the double-stranded cDNA using the MEGAscript T7 kit (Ambion; Thermo Fisher Scientific, Inc.). Aliquots of each reaction mixture were electrophoresed on a $1 \%$ agarose gel to determine the cRNA yield. Subsequently, the cRNA was purified using the RNeasy Mini protocol. Finally, fluorescent cDNA probes were obtained by reverse transcription from the cRNA using the CyScribe First-Strand cDNA labeling kit (GE Healthcare Life Sciences, Piscataway, NJ, USA) with cyanine-3-cytidine triphosphate.

Gene expression profiling and data analysis. The labeled cRNAs were hybridized onto the microarray. Subsequent to washing, the arrays were scanned by the Agilent Scanner G2505C (Agilent Technologies, Inc.). GeneSpring feature extraction software (version 10.7.1.1; Agilent Technologies, Inc.) was used to analyze array images for raw data. GeneSpring was employed to finish the basic analysis with the raw data. The raw data were normalized with the quantile algorithm. The probes with at least $100 \%$ of the values in any 1 out of all conditions with flags in 'Detected' were selected for further data analysis. Differentially expressed genes were then identified through fold changes in addition to the P-values, which were calculated with the t-test. The threshold set for up- and downregulated genes was a fold change $\geq 2.0$ and $\mathrm{P} \leq 0.05$. Subsequently, gene ontology (GO) analysis and Kyoto Encyclopedia of Genes and Genomes analysis were applied to determine the roles of these differentially expressed mRNAs.
Table I. Genes involved in the 'Cytokine-cytokine receptor interaction' pathway with associated P-values acquired from the microarray result.

\begin{tabular}{lcc}
\hline Gene name & P-value & Regulation \\
\hline $\begin{array}{l}\text { V-kit Hardy-Zuckerman 4 feline } \\
\text { sarcoma viral oncogene homolog }\end{array}$ & 0.001781 & Down \\
Interleukin 7 receptor & 0.002597 & Down \\
$\begin{array}{l}\text { Interleukin 28 receptor } \alpha \\
\text { (interferon, } \lambda \text { receptor) }\end{array}$ & 0.013984 & $\mathrm{Up}$ \\
Interleukin 26 & & \\
Chemokine (C-X-C motif) receptor 3 & 0.007562 & $\mathrm{Up}$ \\
Chemokine (C-C motif) receptor 9 & $3.26 \mathrm{E}-04$ & $\mathrm{Up}$ \\
Chemokine (C-C motif) receptor 8 & $6.58 \mathrm{E}-04$ & Down \\
Chemokine (C-C motif) receptor 4 & 0.003523 & $\mathrm{Up}$ \\
Chemokine (C-C motif) receptor 1 & 0.004038 & Down \\
CD40 ligand & $2.68 \mathrm{E}-05$ & $\mathrm{Up}$ \\
\hline
\end{tabular}

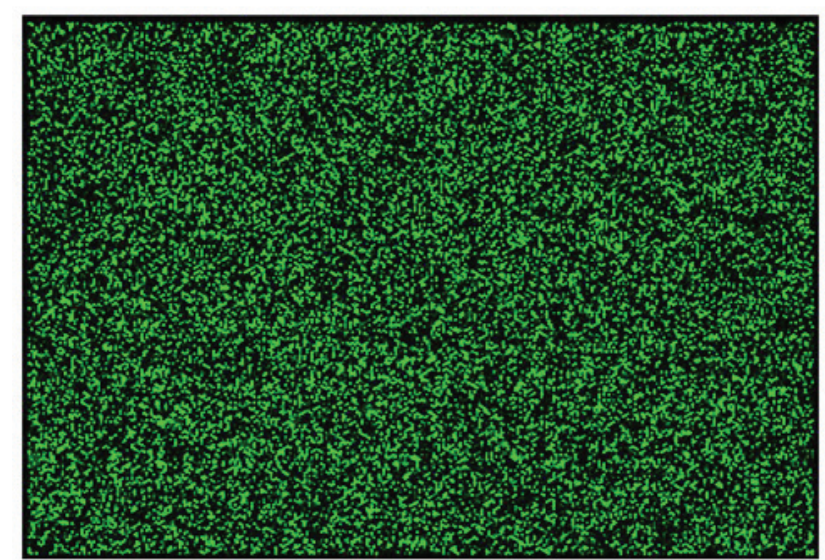

Figure 1. Each experiment produced images that were uniform and clear without marginal effects or scratches, which reflects a good microarray hybridization condition.

Hierarchical clustering was additionally conducted in order to display the gene expression patterns among the samples.

\section{Results}

Microarray scan image. Microarray scan images broadly reflect the microarray hybridization conditions. The clearer and more uniform the images are, the better the hybridization conditions are (Fig. 1).

Scatter plot. The scatter plot is commonly used to assess the variation in RNA expression variation between two samples. Each point of the scatter plot represents a probe on the chip, and the position of the point in the two-dimensional plane is determined by the $\mathrm{X}$-axis and $\mathrm{Y}$-axis coordinates. The signal values of six samples were normalized ( $\log 2$-scaled). All six samples were compared with one another, to generate the matrix plot (Fig. 2). 


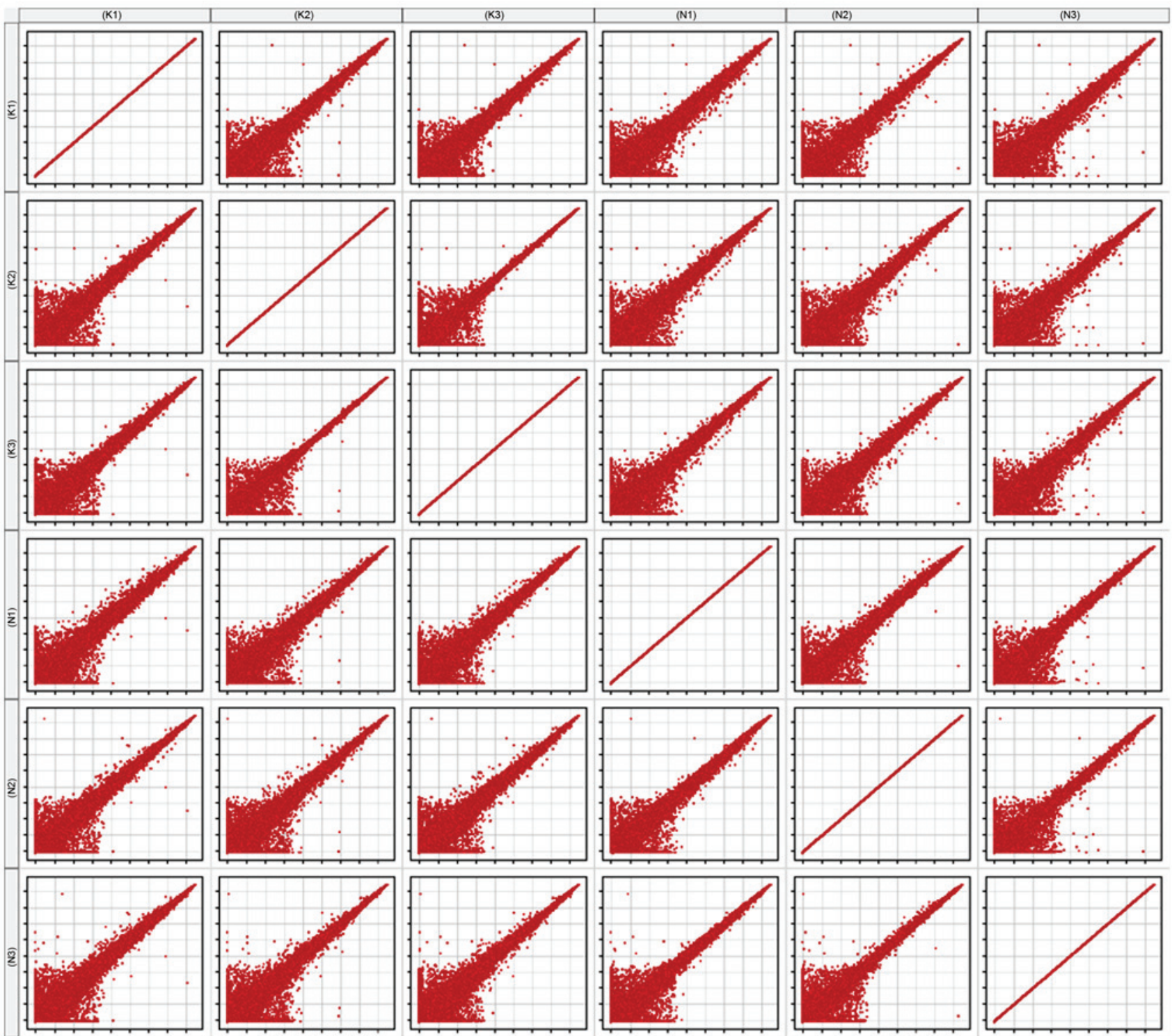

Figure 2. The points above the red lines represent upregulated genes (fold change $>2$ ), whereas points below the red lines represent downregulated genes (fold change $>2$ ).

Gene expression alteration. Of the detected genes, 387 genes were differentially expressed, including 108 upregulated genes and 279 downregulated genes. Hierarchical clustering was performed to highlight the differences in gene expression (Fig. 3).

GO analysis and pathway analysis. GO analysis demonstrated that the differentially expressed genes involve several biological processes, including cell adhesion, the immune response and proteolysis. The components of differential genes were predominantly located in the cell membrane, and the molecular functions included receptor activity, calcium ion binding and G-protein coupled receptor activity. The top 12 significant biological processes (Fig. 4A), cell components (Fig. 4B) and molecular functions (Fig. 4C) are presented in Fig. 4.

Pathway analysis indicated that 10 differentially expressed genes $(23.3 \%)$ are involved in cytokine-cytokine receptor interaction (Table I). The top twelve significant signaling pathways are presented in Fig. 5.

\section{Discussion}

The Golli-MBP protein is distributed in the myelin sheath of the nervous system, and additionally in T cells, macrophages and B cells (3). Upon further study, understanding of the structure and function of proteins is increased. Golli expression has been previously observed to be increased in the lymph nodes of mice with relapsing experimental autoimmune encephalomyelitis (EAE) (6). In heterozygous Golli-MBP knockout mice, EAE relapses were observed to be reduced (7). Previous studies have identifed that in addition to acting as an autoantigen in the pathogenesis of autoimmune diseases, Golli-MBP can be combined with stromal interaction molecule 1 to negatively regulate the proliferation and activation of $\mathrm{T}$ cells, via a mechanism involving the modulation of calcium homeostasis, which is known to serve an important role in the immune system $(4,5)$.

It has been identified that the destruction of MBP correlates with some autoimmune diseases. The cleavage 


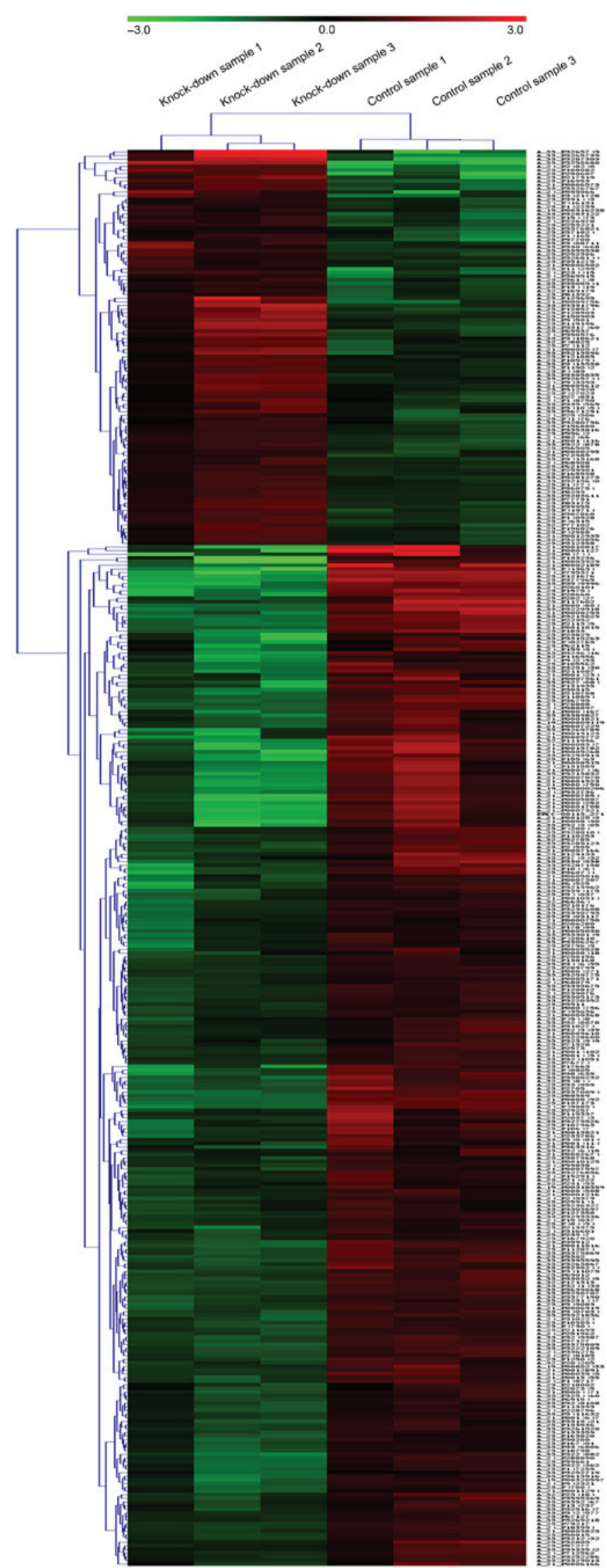

Figure 3. Heat map of differentially expressed genes between 6 samples. Each row represents a probe and each column represents a sample. The color indicates the level of mRNA expression: Green, lower expression; red, higher expression; black, no expression changes. The Golli-myelin basic protein gene knock-down samples were clustered together and clearly separated from three normal samples.
A

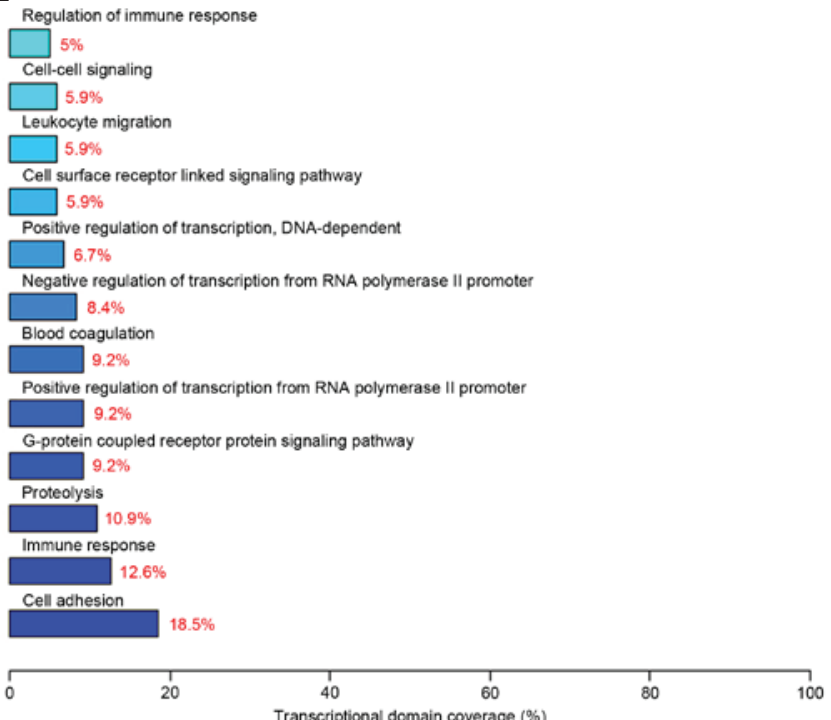

B

Collagen type I

$0.7 \%$

Cytoskeletal part

| $0.7 \%$

Immunological synapse

$1.4 \%$

Acrosomal vesicle

] $2.8 \%$

Ruffle membrane

$\square^{2.8 \%}$

Extracell

$4.2 \%$

Proteinaceous extracellular matrix

$4.9 \%$

External side of plasma membran

$4.9 \%$

Integral to plasma membrane

$17.5 \%$

Extracellular region

$28.7 \%$

Integral to membrane

Plasma membrane $51.7 \%$

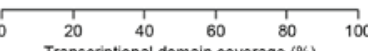

C

Integrin binding

口 $3.4 \%$

Metallopeptidase activity

$\square_{4.2 \%}$

Metalloendopeptidase activity

$\square .2 \%$

Chemokine receptor activity

$\square .2 \%$

Actin binding

$\square 6.8 \%$

$\square_{\text {Receptor binding }}^{6.8 \%}$

$\square 6.8 \%$

$\square .8 \%$
Peptidase activity

\begin{tabular}{l} 
Peptidase \\
$\square$ \\
\hline
\end{tabular}

Protein homodimerization activity

$11 \%$

equence-specific DNA binding

$12.7 \%$

G-protein coupled receptor activity

$14.4 \%$

Calcium ion binding

$15.3 \%$

Receptor activity
$23.7 \%$

\begin{tabular}{llllll}
\hline & 1 & 1 & 1 & 1 & 100
\end{tabular}

Figure 4. The top twelve significant (A) biological process, (B) cell components and $(\mathrm{C})$ molecular functions identified by gene ontology analysis.

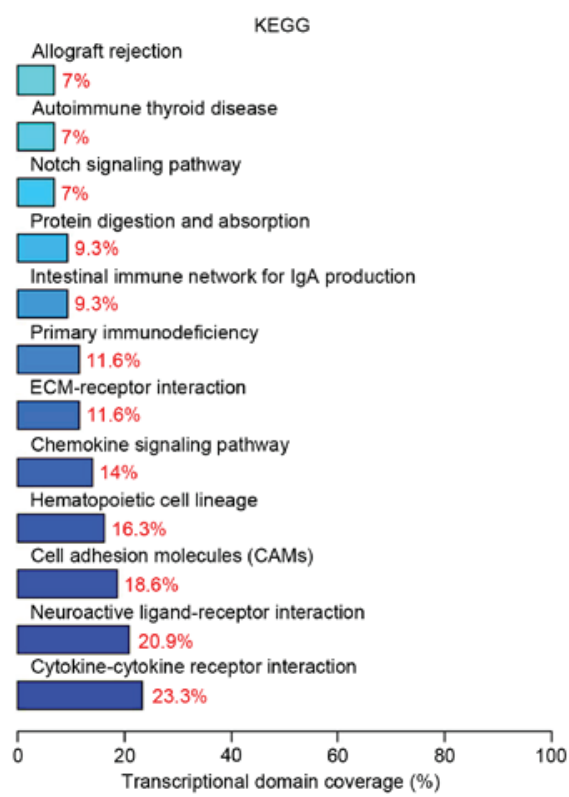

Figure 5. The top twelve significant signaling pathways. 
of MBP has been demonstrated to lead to the generation of immunogenic fragments and demyelination in EAE in addition to human multiple sclerosis $(8,9)$. Previous studies have observed that Golli-MBP exhibited significantly increased expression in oral lichen planus (OLP) than in normal controls, A strong and negative correlation between Golli-MBP and T-helper (Th) 1/Th2 gene expression was observed in peripheral blood mononuclear cells (PBMCs) of patients with OLP (10). This implies that Golli-MBP may influence cytokine gene expression in PBMCs. However, it remains a challenge to explain the association between the abnormal expression of Golli-MBP and the disruption of the equilibrium of Th1/Th2 in patients with OLP. Therefore, the study of alterations in gene expression in the Jurkat $\mathrm{T}$ cell line following downregulation of the Golli-MBP gene may aid in further investigation of whether other genes are involved in influencing $\mathrm{T}$ lymphocyte function together with Golli-MBP.

In the current study, DNA microarray was applied and the results indicated that 387 genes were differentially expressed, including 108 upregulated genes and 279 downregulated genes. GO analysis indicated that the differentially expressed genes involved several biological processes, including cell adhesion, the immune response and the G-protein coupled receptor protein signaling pathway. Pathway analysis demonstrated that the majority of the differentially expressed genes served a role in cytokine-cytokine receptor interaction, the chemokine signaling pathway and cell adhesion molecules. Chemokine receptors were involved in several signaling pathways, and it was hypothesized that Golli-MBP may serve a role in the T lymphocyte signal transduction pathway via a chemokine network, which affects autoimmune diseases.

The current study identified that chemokine (C-X-C motif) receptor (CCR)1, CCR8 and CCR9 were downregulated, while CXCR3 and CCR4 were upregulated. Chemokines are a large family of small proteins and act on the G-protein coupled receptor superfamily. Traditionally, chemokines and their receptors are divided into four families $(\mathrm{CXC}$, $\mathrm{CC}, \mathrm{C}$ and $\mathrm{CX} 3 \mathrm{C}$ ) based on the pattern of cysteine residues in the ligands (11). It has been previously reported that chemokine and chemokine receptors are predominantly associated with the chemotaxis of inflammatory cells and can additionally regulate the activation of immune cells, the secretion of cytokines, cell adhesion, cytotoxic effects, hematopoietic progenitor cell growth and angiogenesis (12). Numerous previous studies have confirmed that the chemokine receptor is closely associated wtih the pathogenesis of human immune-associated diseases, including multiple sclerosis (13), systemic lupus erythematosus (14), asthma (15) and OLP, which is a chronic inflammatory disease with small potential to become malignant (16). The etiology of OLP remains unclear, however it has been previously reported to be a T cell-mediated autoimmune reaction (17). Hu et al (18) reported that serum levels of CCL5 and the percentage of $\mathrm{CCR} 5^{+} \mathrm{CD} 4^{+} \mathrm{T}$ cells was increased in patients with OLP. Chemokine (C-C motif) ligand 5 and CCR1 have been additionally observed to be expressed in OLP and are associated with mast cell migration (19). The chemokine system is a complex immune regulatory network, and further research into the chemokines and their associations is required in order to better understand the role of chemokines in human immune-associated diseases.

Subsequent to knockdown of Golli-MBP, the mechanism regulating the alterations in the biological characteristics in Jurkat cells appeared complex and remains unclear. It is suggested that this may involve numerous types of functional proteins, and metabolic and signaling pathways. However, chemokine receptors were significantly correlated factors that may aid in the investigation of the molecular and biological mechanisms of Golli-MBP in the signal transduction pathway of T-cell-associated autoimmune diseases.

\section{Acknowledgements}

The current study was supported by the National Natural Science Foundation of China (grant nos. 81170961 and 81470748) and a project funded by the Priority Academic Program Development of Jiangsu Higher Education Institutions (grant no. PAPD 2014-37).

\section{References}

1. Feng JM: Minireview: Expression and function of golli protein in immune system. Neurochem Res 32: 273-278, 2007.

2. Campagnoni AT, Pribyl TM, Campagnoni CW, Kampf K, Amur-Umarjee S, Landry CF, Handley VW, Newman SL, Garbay B and Kitamura K: Structure and developmental regulation of Golli-mbp, a 105-kilobase gene that encompasses the myelin basic protein gene and is expressed in cells in the oligodendrocyte lineage in the brain. J Biol Chem 268: 4930-4938, 1993.

3. Pribyl TM, Campagnoni CW, Kampf K, Kashima T, Handley VW, McMahon J and Campagnoni AT: The human myelin basic protein gene is included within a 179-kilobase transcription unit: Expression in the immune and central nervous systems. Proc Natl Acad Sci USA 90: 10695-10699, 1993.

4. Feng JM, Fernandes AO, Campagnoni CW, Hu YH and Campagnoni AT: The golli-myelin basic protein negatively regulates signal transduction in $\mathrm{T}$ lymphocytes. J Neuroimmunol 152: 57-66, 2004

5. Feng JM, Hu YK, Xie LH, Colwell CS, Shao XM, Sun XP, Chen B, Tang $\mathrm{H}$ and Campagnoni AT: Golli protein negatively regulates store depletion-induced calcium influx in T cells. Immunity 24: 717-727, 2006.

6. Slavin AJ, Maron R and Weiner HL: Mucosal administration of IL-10 enhances oral tolerance in autoimmune encephalomyelitis and diabetes. Int Immunol 13: 825-833, 2001.

7. Voskuhl RR, Pribyl TM, Kampf K, Handley V, Liu HB, Feng J, Campagnoni CW, Soldan SS, Messing A and Campagnoni AT: Experimental autoimmune encephalomyelitis relapses are reduced in heterozygous golli MBP knockout mice. J Neuroimmunol 139: 44-50, 2003.

8. Nagasato K, Farris RW 2nd, Dubois-Dalcq M and Voskuhl RR: Exon 2 containing myelin basic protein (MBP) transcripts are expressed in lesions of experimental allergic encephalomyelitis (EAE). J Neuroimmunol 72: 21-25, 1997.

9. Shiryaev SA, Savinov AY, Cieplak P, Ratnikov BI, Motamedchaboki K, Smith JW and Strongin AY: Matrix metalloproteinase proteolysis of the myelin basic protein isoforms is a source of immunogenic peptides in autoimmune multiple sclerosis. PloS One 4: e4952, 2009.

10. Ding M, Zeng J, Sroussi H, Yu J, Xu J, Cheng X and Fan Y: Interactions between Golli-MBP and Th1/Th2 cytokines in patients with oral lichen planus. Oral Dis 20: 205-211, 2014.

11. Allen SJ, Crown SE and Handel TM: Chemokine: Receptor structure, interactions and antagonism. Annu Rev Immunol 25: 787-820, 2007.

12. Bennett LD, Fox JM and Signoret N: Mechanisms regulating chemokine receptor activity. Immunology 134: 246-256, 2011.

13. Cheng W and Chen G: Chemokines and chemokine receptors in multiple sclerosis. Mediators Inflamm 2014: 659206, 2014. 
14. Yu SL, Kuan WP, Wong CK, Li EK and Tam LS: Immunopathological roles of cytokines, chemokines, signaling molecules and pattern-recognition receptors in systemic lupus erythematosus. Clin Dev Immunol 2012: 715190, 2012.

15. Siddiqui S, Secor ER Jr and Silbart LK: Broncho-alveolar macrophages express chemokines associated with leukocyte migration in a mouse model of asthma. Cell Immunol 281: 159-169, 2013.

16. Eisen D: The clinical features, malignant potential and systemic associations of oral lichen planus: A study of 723 patients. J Am Acad Dermatol 46: 207-214, 2002.
17. Mattila R, Ahlfors E and Syrjänen S: CD27 and CD38 lymphocytes are detected in oral lichen planus lesions. Oral Surg Oral Med Oral Pathol Oral Radiol Endod 111: 211-217, 2011.

18. Hu JY, Zhang J, Cui JL, Liang XY, Lu R, Du GF, Xu XY and Zhou G: Increasing CCL5/CCR5 on CD4 ${ }^{+} \mathrm{T}$ cells in peripheral blood of oral lichen planus. Cytokine 62: 141-145, 2013.

19. Zhao ZZ, Sugerman PB, Walsh LJ and Savage NW: Expression of RANTES and CCR1 in oral lichen planus and association with mast cell migration. J Oral Pathol Med 31: 158-162, 2002. 